\title{
Systematic Review \\ Remote Monitoring of Chronic Critically Ill Patients after Hospital Discharge: A Systematic Review
}

\author{
Dmitriy Viderman ${ }^{1}$, Elena Seri ${ }^{2}$, Mina Aubakirova ${ }^{3}$, Yerkin Abdildin ${ }^{4}\left(\mathbb{D}\right.$, Rafael Badenes $^{5, *}$ \\ and Federico Bilotta ${ }^{2}$ (D)
}

check for updates

Citation: Viderman, D.; Seri, E.; Aubakirova, M.; Abdildin, Y.; Badenes, R.; Bilotta, F. Remote Monitoring of Chronic Critically Ill Patients after Hospital Discharge: A Systematic Review. J. Clin. Med. 2022, 11, 1010. https://doi.org/10.3390/ jcm 11041010

Academic Editors: Morgan Broggi, M. Alparslan Turan and Hailin Zhao

Received: 30 November 2021

Accepted: 11 February 2022

Published: 15 February 2022

Publisher's Note: MDPI stays neutral with regard to jurisdictional claims in published maps and institutional affiliations.

Copyright: (C) 2022 by the authors. Licensee MDPI, Basel, Switzerland. This article is an open access article distributed under the terms and conditions of the Creative Commons Attribution (CC BY) license (https:// creativecommons.org/licenses/by/ $4.0 /)$.
1 Department of Anesthesiology and Intensive Care, Nazarbayev University School of Medicine, Nur-Sultan 010000, Kazakhstan; dmitriy.viderman@nu.edu.kz

2 Department of Anesthesiology, Critical Care and Pain Medicine, Policlinico Umberto I, "Sapienza" University of Rome, 00185 Rome, Italy; eliseri2499@gmail.com (E.S.); bilotta@tiscali.it (F.B.)

3 Department of Biomedical Sciences, Nazarbayev University School of Medicine, Nur-Sultan 010000, Kazakhstan; mina.aubakirova@nu.edu.kz

4 School of Engineering and Digital Sciences, Nazarbayev University, Nur-Sultan 010000, Kazakhstan; yerkin.abdildin@nu.edu.kz

5 Department of Anesthesiology and Surgical-Trauma Intensive Care, Hospital Clínic Universitari, University of Valencia, 46010 Valencia, Spain

* Correspondence: rafaelbadenes@gmail.com

\begin{abstract}
Background: Over the past few decades, critical care has seen many advancements. These advancements resulted in a considerable increase in the prevalence of chronically critically ill patients requiring prolonged medical care, which led to a massive increase in healthcare utilization. Methods: We performed a search for suitable articles using PubMed and Google Scholar from the inception of these databases to 15 May 2021. Results: Thirty-four articles were included in the review and analyzed. We described the following characteristics and problems with chronic critically ill patient management: the patient population, remote monitoring, the monitoring of physiological parameters in chronic critically ill patients, the anatomical location of sensors, the barriers to implementation, and the main technology-related issues. The main challenges in the management of these patients are (1) the shortage of caretakers, (2) the periodicity of vital function monitoring (e.g., episodic measuring of blood pressure leads to missing important critical events such as hypertension, hypotension, and hypoxia), and (3) failure to catch and manage critical physiological events at the right time, which can result in poor outcomes. Conclusions: The prevalence of critically ill patients is expected to grow. Technical solutions can greatly assist medical personnel and caregivers. Wearable devices can be used to monitor blood pressure, heart rate, pulse, respiratory rate, blood oxygen saturation, metabolism, and central nervous system function. The most important points that should be addressed in future studies are the performance of the remote monitoring systems, safety, clinical and economic outcomes,
\end{abstract} as well as the acceptance of the devices by patients, caretakers, and healthcare professionals.

Keywords: critical care; remote monitoring; remote neurological monitoring; glucose monitoring; chronic critical illness; long-term care

\section{Introduction}

Over the past decades, critical care has made significant advancements that have improved the outcomes of critically ill patients. Unfortunately, these advancements contributed to a considerable increase in the prevalence of chronic critical illness requiring prolonged medical care, including mechanical ventilation, which has led to a massive increase in healthcare utilization [1]. 
Several definitions of chronic critical illness (CCI) have been proposed. One of the most accepted is the following: "a disease state which affects intensive care patients who have survived an initial insult but remain dependent on intensive care for a protracted period, neither dying nor recovering" [2].

Although the exact prevalence of CCI patients is difficult to estimate, it was calculated to be as high as 250,000 patients in the United States alone, and rapidly growing [3,4]. It is expected to grow $50-100 \%$ in every upcoming decade [3,4]. Healthcare spending related to chronic critical illness has been estimated to exceed $\$ 20$ billion and is expected to rise further [1]. Furthermore, readmission rates within one year after hospital discharge have exceeded $40 \%$ [5]. Many patients who are discharged to long-term care facilities are unable to be adequately rehabilitated in order to return home within 6 months and are usually institutionalized until death [6]. An additional challenge for CCI patients is life quality and expectancy given that fewer than $12 \%$ of chronically critically ill patients were alive and independent one year after their acute illness [7-10]. The clinical course and management strategy of chronic critical illness are different from those of acute critical illness. Throughout the course of the chronic critical illness, the clinical status usually fluctuates slowly [11]. Improvement of condition and organ-system function takes place slowly and usually takes weeks or months to occur [11]. It is also important to ascertain that although chronic critical illness is a chronic process, it can include rapid acute events such as hemodynamic instability, heart failure, pneumonia, and sepsis that require an escalation of management [11]. The unpredictable clinical trajectory of chronic critical illness requires caregivers to have unique skills sets that should ideally combine rehabilitation, emergency, and sometimes critical care skills. Occasionally it is not possible to observe CCI patient's conditions on a constant basis; therefore, technological support that monitors vital functions, detects functional deterioration, and possibly replaces or supports vital functions is required [11].

One of the central issues in the management of CCI patients after their discharge from the hospital is the absence of timely and continuous monitoring of even the most basic physiological parameters, and the lack of immediate correction and treatment when needed. After discharge from the hospital, these patients frequently have unstable vital functions [11]. Moreover, basic physiological parameters, such as blood pressure, pulse, respiratory rate, consciousness, and oxygen saturation, are measured episodically; therefore, important pathological events can be frequently missed. Failure to recognize these events can result in life-threatening complications and make all previous attempts by the medical team to manage the patient futile. Therefore, for more organized management, the creation of specialized facilities was proposed [11].

Remote patient monitoring suggests a promising direction in healthcare that could help in the diagnosis and treatment of patients remotely using sensor devices, telecommunication, and information technology solutions for the management of chronic critical illness. These solutions collect medical data directly from the patient and transmit the data to the caregivers and healthcare providers for interpretation and recommendations [12].

Remote patient monitoring may help to better control common conditions, their complications, and life-threatening events (e.g., metabolic, respiratory, cardiovascular, and neurological), therefore reducing the risk of hospitalization and mortality and improving the overall quality of life and services.

The purpose of this systematic review is to report evidence on the possible applications of wireless remote monitoring of chronic critical illness patients after hospital discharge with the aim of indicating a potential pathway for future research to develop evidencebased recommendations using cutting-edge remote monitoring technologies.

\section{Materials and Methods}

This systematic review was performed in accordance with the Preferred Reporting Items for Systematic Reviews and Meta-Analyses (PRISMA) statement [13]. The study was 
registered and accepted into the international prospective register of systematic review databases (PROSPERO registration number: CRD42021255515) [14].

We developed a protocol for matching publications which was established and approved by the research group. We predefined our research subtopics and performed a systematic review to summarize the current state of application of wireless remote monitoring of chronically critically ill patients after hospital discharge.

The steps in our evidence search and synthesis include: (1) Identification of relevant publications; (2) Data extraction; (3) Data analysis, aggregation, and summarization of the results; (4) Synthesis of the existing data; (5) Identifying the implications of the study findings; (6) Detailing the existing clinical gaps; (7) and Synthesis of conclusions.

We defined the scope of this review as all available articles reporting wearable technologies and sensors that might be used for chronically critically ill patients after discharge from the hospital.

Article selection: Articles were included in this systematic review if they mentioned the following: (1) 18 years and older; (2) Clearly described methodology of the study; (3) Applications of wireless remote monitoring of chronic critically ill patients after hospital discharge that can potentially be used for vital function monitoring (cardiovascular, respiratory, nervous system, temperature); (4) and Vital function monitoring.

Articles were excluded from the study if: (1) they did not clearly describe the study methodology; (2) they were animal studies; (3) and if they were pediatric studies ( $<18$ years old).

Settings: Any healthcare setting (medical centers, hospitals, clinics).

Types of study to be included: all types of studies and reports should be included in accordance with inclusion. Both retrospective and prospective studies were considered.

Search methods: We performed a search for suitable articles using PubMed and Google Scholar from the inception of these databases to 15 May 2021. The searches included the following terms and their combinations: "chronic critical illness", "critical illness", "remote monitoring," "outcome," and "monitoring". We searched the journals and references for all articles relevant to the study. Ethical approval and patient consent were not required. Since remote patient monitoring was not well studied in the chronically ill patient population after their discharge from the hospital, we identified the main syndromes and symptoms commonly seen in chronic critical illness (Figure 1) and searched for technologies that could potentially be used in this patient population. The following information was extracted: reference, first author, year of publication, study goals, study type, targeted population, age, gender, sample size, diagnosis, comorbidities, type of device, monitoring parameter (analysis), electroencephalogram (EEG), electrocardiogram (ECG), glucose level, outcomes, location of sensors, data processing, artificial intelligence method, performance of the models, and implementation barriers.

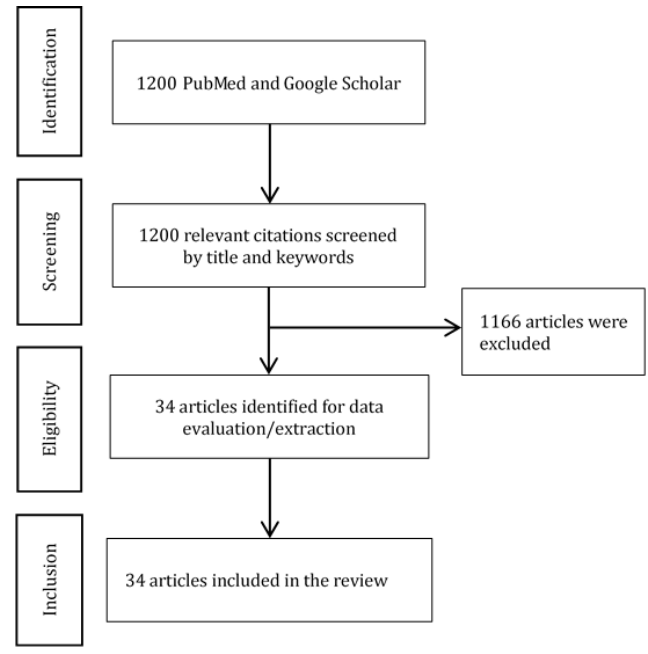

Figure 1. PRISMA diagram. 


\section{Results}

\subsection{Study Characteristics}

A literature search yielded 1200 publications. After the removal of duplicates and the selection of studies that met the inclusion criteria, 34 articles were included in the review and analyzed (Figure 1) [9,15-51]. Data on the study characteristics, including author, year of publication, country, study objective and design, study population and sample size, patient age and gender distribution, type of device, location of sensor, parameter of monitoring, issues with devices and barriers to implementation, as well as study conclusions and findings are summarized in Table 1.

\subsection{Patient Population}

Chronically critically ill patients present a wide variety of symptoms and syndromes and may require medical and surgical treatments, including: (1) remote monitoring of infections, hemodynamics, sepsis, and pain; (2) remote monitoring of surgical care (neurosurgical, orthopedic, spinal, vascular, abdominal, transplant); (3) remote monitoring of patients undergoing acute or chronic neurologic care (including dementia); (4) remote monitoring of patients receiving opioids and other centrally acting central nervous system suppressants.

\subsection{Remote Monitoring of Physiological Parameters in Chronic Critical Ill Patients}

Since one of the most important issues in the management of such patients is the failure to continuously monitor physiological functions, the missing of critical events and late responses to derangements can lead to complications. The following physiological parameters have been monitored and reported in the published studies: non-invasive blood pressure, pulse, respiratory rate, $\mathrm{SpO} 2$, skin temperature, electrocardiogram, continuous noninvasive blood pressure, respiratory rate, pulse oximetry, temperature, body posture, fall detection, activity, step count, and ambulation (Table 1) [9,15-47].

\subsection{Anatomical Location of the Sensor}

The most common anatomical locations for sensors were the chest (sensors for respiratory rate, ECG), wrist, thumb (sensor for $\mathrm{SpO} 2$ and $\mathrm{BP}$ ), thigh, calf, and lower arm.

Patient-related issues:

1. Devices were described as heavy, bulky, uncomfortable to wear, difficult to wear while performing activities, difficult to wear while eating, difficult to wear while washing hands; 2 . Patients reported anxiety over possible injury and pressure sores; 3 . Patients were concerned that remote monitoring will replace face-to-face interaction with nurses [7].

Technical issues of using remote monitoring devices:

1. Issues with responsiveness of the screen to input, robustness, and ease of cleaning the device; 2 . Artifacts caused by connection failure [4], motion of the sensors, patient movements, and need for calibration of the physiological parameters such as blood pressure; 3. A large amount of data generated each day by a wearable device; 4 . The digital patch was reported to be unsuitable for patients with atrial fibrillation [48]; 5. Monitoring devices deliver falsely reassuring data that may reduce the attention that patients require [48]; 6 . The differences between vital sign patches and manual measurements of vital signs were out of acceptable limits [49]; 7. Signal quality can be affected by many factors such as inappropriate sensor-skin coupling due to device malposition, pressure on skin, ambient light, and biological factors and motions [50]; 8. The devices for remote monitoring require the development and improvement of interoperability standards to assist device connectivity and the integration of a monitor into medical settings [6]; 9. Data loss due to technical issues [49]; 10. Large volume of data related to cardio-respiratory function (heart rate/rhythm and respiratory rate cancelled by the system) [48,52]; 11. Redundant amount of data can produce "false-positive" outcomes and, therefore, should be double-checked carefully to warrant use of new technologies and approaches in trauma care [53]. 
Table 1. Description of characteristics of included studies.

\begin{tabular}{|c|c|c|c|c|c|c|c|c|c|}
\hline \# & $\begin{array}{l}\text { First Author, Year, } \\
\text { Country, Reference }\end{array}$ & $\begin{array}{l}\text { Objective (Study } \\
\text { Type) }\end{array}$ & $\begin{array}{l}\text { Targeted Population } \\
(n)\end{array}$ & Gender (M/F, \%) & Age & $\begin{array}{l}\text { Device (Location of } \\
\text { Sensor) }\end{array}$ & $\begin{array}{l}\text { Parameter of } \\
\text { Monitoring }\end{array}$ & $\begin{array}{l}\text { Problems with } \\
\text { Devices/Barriers to } \\
\text { Implementation }\end{array}$ & Outcomes/Findings \\
\hline 1 & $\begin{array}{l}\text { Prgomet et al., 2016, } \\
\text { Australia [15] }\end{array}$ & $\begin{array}{c}\text { Clinical staff } \\
\text { perceptions to } \\
\text { monitoring practices } \\
\text { (mixed methods) }\end{array}$ & $\begin{array}{l}\text { Physicians, nurses } \\
\quad(n=106)\end{array}$ & $12.5 / 87.5$ & $\begin{array}{c}18-44=85 \% \\
45+=10 \%\end{array}$ & ViSi Mobile (wrist) & $\begin{array}{c}\text { BP, pulse, RR, } \mathrm{SpO} 2, \\
\text { T, ECG }\end{array}$ & $\begin{array}{l}\text { Inconvenience; } \\
\text { technical issues; } \\
\text { substitution of } \\
\text { nurses with devices; } \\
\text { false alarms } \\
\end{array}$ & $\begin{array}{l}\text { Positive expectations of } \\
\text { CM on care improvemen }\end{array}$ \\
\hline 2 & $\begin{array}{l}\text { Weller et al., 2017, } \\
\text { USA [16] }\end{array}$ & $\begin{array}{l}\text { Clinical outcomes } \\
\text { under standard } \\
\text { versus continuous } \\
\text { VS monitoring with } \\
\text { low alarm rates } \\
\text { (case-control) }\end{array}$ & $\begin{array}{l}\text { Older neuro- and } \\
\text { neurosurgery } \\
\text { patients }(n=736)\end{array}$ & $\begin{array}{l}\text { I: } 54 / 46 \\
\text { C: } 52 / 48\end{array}$ & $\begin{array}{l}\text { I: } 60.5(14.7) \\
\text { C: } 60.1(15.5)\end{array}$ & $\begin{array}{l}\text { ViSi Mobile (not } \\
\text { reported) }\end{array}$ & BP, HR, RR, SpO2 & $\begin{array}{l}\text { Possibility of false } \\
\text { alarms or } \\
\text { overlooking real } \\
\text { deterioration }\end{array}$ & $\begin{array}{l}\text { CM was effective in } \\
\text { detection of VS changes } \\
\text { at a low alarm rate }\end{array}$ \\
\hline 3 & $\begin{array}{l}\text { Verrillo et al., 2018, } \\
\text { USA [17] }\end{array}$ & $\begin{array}{l}\text { Effects of using } \\
\text { routine versus } \\
\text { continuous VS } \\
\text { surveillance } \\
\text { (before-after) }\end{array}$ & $\begin{array}{l}\text { Orthopedics, trauma } \\
(n=864 ; \mathrm{I}=422, C= \\
427, \text { Survey }=15)\end{array}$ & $\begin{array}{c}\text { I: } 54 / 46, \\
\text { C: } 58 / 42 \text {, Survey: } \\
0 / 100\end{array}$ & $\begin{array}{l}\text { I: } 54.45(52.8-56.1) \\
\text { C: } 51.44(49.7-53.2) \\
\text { S: up to } 29\end{array}$ & $\begin{array}{l}\text { ViSi Mobile (Chest, } \\
\text { wrist, thumb) }\end{array}$ & $\begin{array}{c}\text { HR, } \\
\text { BP, RR, SpO2, T }\end{array}$ & None reported & $\begin{array}{c}\text { CM allowed for } \\
\text { improved detection of } \\
\text { state exacerbation, lower } \\
\text { complication rates, } \\
\text { similar incidence of RRTs } \\
\text { reduced ICU requiremen }\end{array}$ \\
\hline 4 & $\begin{array}{l}\text { Weenk et al., 2017, the } \\
\text { Netherlands [18] }\end{array}$ & $\begin{array}{l}\text { VS measurements by } \\
\text { nurses versus two } \\
\text { CM devices, and } \\
\text { experience } \\
\text { perceptions (mixed } \\
\text { methods) }\end{array}$ & $\begin{array}{l}\text { Internal medicine } \\
\text { (sepsis, arthritis, BP } \\
\text { control) and surgical } \\
\text { patients }(n=20)\end{array}$ & $65 / 35$ & $\begin{array}{l}49.9 \text { (13.4), range } \\
33-82\end{array}$ & $\begin{array}{c}\text { ViSi Mobile (Chest, } \\
\text { wrist, thumb), } \\
\text { HealthPatch (Chest, } \\
\text { wrist) }\end{array}$ & $\begin{array}{c}\text { Visi Mobile: ECG, } \\
\text { HR, SpO2, RR, T, } \\
\text { and BP } \\
\text { HealthPatch: ECG, } \\
\text { HR, HRV, RR, T, } \\
\text { body posture, fall } \\
\text { detection, and } \\
\text { activity }\end{array}$ & $\begin{array}{l}\text { Artefacts due to } \\
\text { technical issues, } \\
\text { body motion, sensor } \\
\text { detachment, and } \\
\text { failure to carry the } \\
\text { mobile device at all } \\
\text { times. Skin irritation; } \\
\text { inconvenience; } \\
\text { detachment from } \\
\text { skin; quick battery } \\
\text { discharge; weak } \\
\text { connectivity; large } \\
\text { amount of data }\end{array}$ & $\begin{array}{l}\text { Consistency in VS } \\
\text { measured by both } \\
\text { devices and manually. } \\
\text { MEWS clinically } \\
\text { significantly differed due } \\
\text { to inconsistent RR } \\
\text { measurements. Artifacts } \\
\text { due to attachment issues } \\
\text { and for undetected } \\
\text { reasons. Positive } \\
\text { attitudes. }\end{array}$ \\
\hline 5 & $\begin{array}{l}\text { Watkins et al., 2015, } \\
\text { USA [19] }\end{array}$ & $\begin{array}{l}\text { Evaluation of VS CM } \\
\text { in hospital settings } \\
\text { (prospective } \\
\text { observational) }\end{array}$ & $\begin{array}{c}\text { Patients and nurses } \\
\text { in medical and } \\
\text { surgical unit }(n=236 \\
\text { patients, } \\
n=24 \text { nurses })\end{array}$ & NA & NA & $\begin{array}{l}\text { ViSi Mobile (Not } \\
\text { reported) }\end{array}$ & $\mathrm{SpO}_{2}, \mathrm{HR}, \mathrm{BP}, \mathrm{RR}$ & $\begin{array}{l}\text { Possibility for } \\
\text { excessive number of } \\
\text { alarms }\end{array}$ & $\begin{array}{l}\text { Feasibility of } \mathrm{CM} \text { at a } \\
\text { reasonable alarm rate }\end{array}$ \\
\hline 6 & $\begin{array}{c}\text { Downey et al., 2018, } \\
\text { UK [20] }\end{array}$ & $\begin{array}{l}\text { Evaluation of VS CM } \\
\text { practicality for } \\
\text { surgery patients } \\
\text { (pilot RCT) }\end{array}$ & $\begin{array}{l}\text { Surgical patients } \\
\quad(n=350)\end{array}$ & $54 / 46$ & $65.2,24-94$ & $\begin{array}{l}\text { SensiumVitals } \\
\text { (Chest) }\end{array}$ & HR, RR, T & $\begin{array}{c}\text { Excessive number of } \\
\text { alerts before } \\
\text { parameter resets. } \\
\text { Various levels of } \\
\text { involvement among } \\
\text { nurses }\end{array}$ & $\begin{array}{c}\text { Faster reception of } \\
\text { antibiotics for sepsis, less } \\
\text { time of hospitalization, } \\
\text { lower 30-day } \\
\text { readmission rates, higher } \\
\text { perception of feeling } \\
\text { comfortable and safe for } \\
\text { the CM group }\end{array}$ \\
\hline
\end{tabular}


Table 1. Cont.

\begin{tabular}{|c|c|c|c|c|c|c|c|c|c|}
\hline$\#$ & $\begin{array}{l}\text { First Author, Year, } \\
\text { Country, Reference }\end{array}$ & $\begin{array}{l}\text { Objective (Study } \\
\text { Type) }\end{array}$ & $\begin{array}{l}\text { Targeted Population } \\
\qquad(n)\end{array}$ & Gender $(M / F, \%)$ & Age & $\begin{array}{l}\text { Device (Location of } \\
\text { Sensor) }\end{array}$ & $\begin{array}{l}\text { Parameter of } \\
\text { Monitoring }\end{array}$ & $\begin{array}{l}\text { Problems with } \\
\text { Devices/Barriers to } \\
\text { Implementation }\end{array}$ & Outcomes/Findings \\
\hline 7 & $\begin{array}{l}\text { Downey et al., 2018, } \\
\text { UK [21] }\end{array}$ & $\begin{array}{l}\text { Patients' perceptions } \\
\text { of in-hospital CM } \\
\text { (Qualitative) }\end{array}$ & $\begin{array}{l}\text { Surgical patients } \\
\qquad(n=12)\end{array}$ & $50 / 50$ & $42-83$ & $\begin{array}{l}\text { SensiumVitals } \\
\text { (Chest) }\end{array}$ & HR, RR, T & $\begin{array}{l}\text { Unpractical and not } \\
\text { comfortable. Worry } \\
\text { that the devices are } \\
\text { not reliable and will } \\
\text { substitute medical } \\
\text { staff }\end{array}$ & $\begin{array}{l}\text { CM perceived as } \\
\text { valuable, especially at } \\
\text { night, but lacking } \\
\text { personal communication } \\
\text { and unable to clarify } \\
\text { health-related } \\
\text { uncertainties }\end{array}$ \\
\hline 8 & $\begin{array}{l}\text { Hernandez-Silveira et al., } \\
\text { 2015, UK [22] }\end{array}$ & $\begin{array}{c}\text { Comparison of } \\
\text { measurements } \\
\text { between CM device } \\
\text { and bedside monitor } \\
\text { (Validation) }\end{array}$ & $\begin{array}{c}\text { Elective surgery (1) } \\
\text { and general ward (2) } \\
\text { patients } \\
(n=61 ; 1=20 ; 2= \\
41)\end{array}$ & $\begin{array}{l}1: 65 / 35 \\
2: 78 / 22\end{array}$ & $1=49(16)$ & $\begin{array}{l}\text { SensiumVitals } \\
\text { (Chest) }\end{array}$ & HR, RR, T & $\begin{array}{c}\text { Not reliable for } \\
\text { patients with atrial } \\
\text { fibrillation. False } \\
\text { negatives may result } \\
\text { in lack of attention }\end{array}$ & $\begin{array}{l}\text { Acceptable consistency of } \\
\text { measurements between } \\
\text { the CM device and } \\
\text { bedside monitor: } 80 \% \text { for } \\
\text { HR and } 50 \% \text { for RR }\end{array}$ \\
\hline 9 & $\begin{array}{c}\text { Hernandez-Silveira et al., } \\
\text { 2015, UK [23] }\end{array}$ & $\begin{array}{l}\text { Demonstration of } \\
\text { practicality of a CM } \\
\text { device in a hospital } \\
\text { (Validation) }\end{array}$ & $\begin{array}{l}\text { Patient simulators } \\
\text { (1); healthy } \\
\text { volunteers (2); } \\
\text { clinical patients } \\
\text { acute (3) }(1=333 ; 2 \text {; } \\
\text { first stage = } 21 \\
\text { second stage }=6 ; 3= \\
41)\end{array}$ & $\begin{array}{c}1,3: \text { NA; } 2: \text { first } \\
\text { stage }=86 / 14 \\
\text { second stage }=83\end{array}$ & $\begin{array}{c}1=\mathrm{NA} ; 2 \text { : first stage } \\
=32.1(6.9), \text { second } \\
\text { stage = } 34.1(11.6) ; \\
3=18-85\end{array}$ & SensiumVital (Chest) & HR, RR, T & $\begin{array}{l}\text { High rate of } \\
\text { rejections in RR data } \\
\text { for clinical patients }\end{array}$ & $\begin{array}{l}\text { Satisfactory agreement } \\
\text { between of } \\
\text { measurements with a } \\
\text { clinically approved } \\
\text { bedside monitor. }\end{array}$ \\
\hline 10 & $\begin{array}{c}\text { Downey et al., 2019, } \\
\text { UK [24] }\end{array}$ & $\begin{array}{c}\text { Validation of } \\
\text { accuracy of HR, RR, } \\
\text { and T measurements } \\
\text { by a CM device } \\
\text { (Validation) }\end{array}$ & $\begin{array}{c}\text { Post-operative } \\
\text { patients }(n=51)\end{array}$ & Not reported & Not reported & $\begin{array}{l}\text { SensiumVitals } \\
\text { (Chest) }\end{array}$ & HR, RR, T & $\begin{array}{l}\text { RR artefacts possibly } \\
\text { due to speaking. } \\
\text { Differences in VS } \\
\text { measurements by } \\
\text { CM device and } \\
\text { manually }\end{array}$ & $\begin{array}{l}\text { Moderate correlations } \\
\text { between measurements } \\
\text { for HR (with large } \\
\text { discrepancies), low } \\
\text { correlations for RR and T }\end{array}$ \\
\hline 11 & $\begin{array}{l}\text { Chan et al., 2013, } \\
\text { USA [25] }\end{array}$ & $\begin{array}{l}\text { Analysis of } \\
\text { performance of a } \\
\text { CM device } \\
\text { (Validation) }\end{array}$ & $\begin{array}{c}\text { Older (1) and } \\
\text { younger }(2) \text { healthy } \\
\text { adults }(n=35 ; 1=15 ; \\
2=10)\end{array}$ & $\begin{array}{l}1: 47 / 53 \\
2: 50 / 50\end{array}$ & $\begin{array}{c}\text { 1: } 70(5), 63-79 \\
\text { 2: } 25(3.6), 18-29\end{array}$ & $\begin{array}{l}\text { Bluetooth Low } \\
\text { Energy (BLE) (Over } \\
\text { ICS } 2 \text { or } 6 \text { or over the } \\
\text { upper sternum) }\end{array}$ & $\begin{array}{l}\mathrm{HR}, \mathrm{HRV}, \mathrm{RR}, \\
\text { posture, steps, falls }\end{array}$ & $\begin{array}{l}\text { Need for } \\
\text { user-friendliness for } \\
\text { wider acceptability }\end{array}$ & $\begin{array}{c}\text { CM devices produce } \\
\text { similar observations as } \\
\text { standard and more bulky } \\
\text { equipment }\end{array}$ \\
\hline 12 & $\begin{array}{c}\text { Izmailova et al., 2019, } \\
\text { USA [26] }\end{array}$ & $\begin{array}{c}\text { Evaluation of } \\
\text { measurements of VS } \\
\text { and physical activity } \\
\text { by two CM devices } \\
\text { (Validation) }\end{array}$ & $\begin{array}{l}\text { Healthy adults } \\
\qquad(n=6)\end{array}$ & $83 / 17$ & $18-55$ & $\begin{array}{l}\text { Actiwatch Spectrum } \\
\text { Pro (A) (wrist); } \\
\text { Vitalconnect } \\
\text { HealthPatch MD } \\
\text { (HP) (left upper } \\
\text { precordium) }\end{array}$ & $\begin{array}{l}\text { A: mobility and } \\
\text { sleep } \\
\text { HP: HR, RR, T }\end{array}$ & $\begin{array}{l}\text { Poor correlation } \\
\text { with hospital } \\
\text { measurements, false } \\
\text { signal of tachycardia, } \\
\text { time-consuming to } \\
\text { double-check }\end{array}$ & $\begin{array}{l}\text { HealthPatch showed a } \\
\text { strong correlation for HR, } \\
\text { but not for RR or T, with } \\
\text { manual measurements. } \\
\text { Actiwatch found } \\
\text { acceptable for physical } \\
\text { activity/sleep } \\
\text { surveillance and for } \\
\text { assistance in interpreting } \\
\text { VS data }\end{array}$ \\
\hline 13 & $\begin{array}{l}\text { Breteler et al., 2018, The } \\
\text { Netherlands [27] }\end{array}$ & $\begin{array}{l}\text { Realiability of HR } \\
\text { and RR } \\
\text { measurements by a } \\
\text { CM device } \\
\text { (Observational } \\
\text { comparisons) }\end{array}$ & $\begin{array}{c}\text { Post-surgery } \\
\text { patients }(n=25)\end{array}$ & $72 / 28$ & $63(57.8-71.5)$ & $\begin{array}{l}\text { HealthPatch MD } \\
\text { (Chest) }\end{array}$ & $\begin{array}{l}\text { ECG, HR, HRV, RR, } \\
\text { T, posture, steps }\end{array}$ & $\begin{array}{l}\text { Missing data due to } \\
\text { unstable battery. } \\
\text { Possible need to } \\
\text { manually delete } \\
\text { artefacts }\end{array}$ & $\begin{array}{l}\text { Accurate measurements } \\
\text { for HR but not for RR }\end{array}$ \\
\hline
\end{tabular}


Table 1. Cont.

\begin{tabular}{|c|c|c|c|c|c|c|c|c|c|}
\hline$\#$ & $\begin{array}{l}\text { First Author, Year, } \\
\text { Country, Reference }\end{array}$ & $\begin{array}{c}\text { Objective (Study } \\
\text { Type) }\end{array}$ & $\begin{array}{l}\text { Targeted Population } \\
\qquad(n)\end{array}$ & Gender (M/F, \%) & Age & $\begin{array}{l}\text { Device (Location of } \\
\text { Sensor) }\end{array}$ & $\begin{array}{l}\text { Parameter of } \\
\text { Monitoring }\end{array}$ & $\begin{array}{l}\text { Problems with } \\
\text { Devices/Barriers to } \\
\text { Implementation }\end{array}$ & Outcomes/Findings \\
\hline 14 & $\begin{array}{l}\text { Selvaraj et al., 2018, } \\
\text { USA [28] }\end{array}$ & $\begin{array}{l}\text { Presentation and lab } \\
\text { validation of a CM } \\
\text { device (Validation) }\end{array}$ & $\begin{array}{l}\text { Healthy volunteers } \\
\qquad(n=57)\end{array}$ & $58 / 42$ & $35(11)$ & VitalPatch (Chest) & $\begin{array}{l}\text { HR, BR, posture, } \\
\text { steps, and falls }\end{array}$ & None reported & $\begin{array}{c}\text { Accurate measurements } \\
\text { of VS and rest-activity } \\
\text { cycles }\end{array}$ \\
\hline 15 & Liu et al., 2014, USA [29] & $\begin{array}{l}\text { Usefulness of a } \\
\text { wireless CM device } \\
\text { in ER for LSI } \\
\text { (Validation) }\end{array}$ & $\begin{array}{l}\text { Code } 2 / 3 \text { trauma } \\
(n=305 ; C=201 \\
I=104)\end{array}$ & $\begin{array}{l}\text { Overall } \\
66 / 34\end{array}$ & $\begin{array}{l}\text { Overall } \\
39(16)\end{array}$ & $\begin{array}{l}\text { The wireless vital } \\
\text { signs monitor } \\
\text { (WVSM) (arm, } \\
\text { thumb) }\end{array}$ & $\begin{array}{c}\text { ECG, } \\
\text { BP, SpO2 }\end{array}$ & $\begin{array}{l}\text { Human error during } \\
\text { attachment to the } \\
\text { patient. Training of } \\
\text { medical staff, } \\
\text { adaptation of } \\
\text { medical settings to } \\
\text { the device }\end{array}$ & $\begin{array}{l}\text { Improvement in LSI } \\
\text { using CM device in ER } \\
\text { settings }\end{array}$ \\
\hline 16 & Liu et al., 2015, USA [30] & $\begin{array}{l}\text { Assessment of VS } \\
\text { data quality of a } \\
\text { wireless CM device } \\
\text { and its ability to } \\
\text { forecast requirement } \\
\text { of LSIs (Cohort) }\end{array}$ & $\begin{array}{l}\text { Code } 2 / 3 \text { trauma } \\
\quad(n=104)\end{array}$ & $79 / 21$ & $40(16)$ & WVSM (arm, thumb) & $\begin{array}{c}\text { HR, BP, } \\
\text { MAP, RR, SpO2, } \\
\text { shock index, pulse } \\
\text { pressure }\end{array}$ & $\begin{array}{l}\text { Possibility for } \\
\text { false-positive } \\
\text { observations }\end{array}$ & $\begin{array}{l}\text { Useful for forecasting LSI } \\
\text { requirement, the majority } \\
\text { of data being high quality }\end{array}$ \\
\hline 17 & $\begin{array}{c}\text { Razjouan et al., 2017, } \\
\text { USA [31] }\end{array}$ & $\begin{array}{l}\text { Effectiveness of a } \\
\text { CM device to predict } \\
\text { risk of fall (Cohort) }\end{array}$ & $\begin{array}{l}\text { Hematology and } \\
\text { oncology }(n=31)\end{array}$ & $45 / 55$ & $59.5(16.1)$ & $\begin{array}{l}\text { Zephyr BioPatch } \\
\text { (Chest) }\end{array}$ & $\begin{array}{l}\text { ECG, RR, } \mathrm{T} \\
\text { 3-dimensional } \\
\text { acceleration }\end{array}$ & None reported & $\begin{array}{l}\text { Risk of fall can be } \\
\text { predicted by monitoring } \\
\text { sleep and activity } \\
\text { patterns and HRV }\end{array}$ \\
\hline 18 & $\begin{array}{c}\text { Boatin et al., 2016, } \\
\text { USA [32] }\end{array}$ & $\begin{array}{l}\text { Usefulness and } \\
\text { patient experiences } \\
\text { of a VS device } \\
\text { (Mixed methods) }\end{array}$ & $\begin{array}{l}\text { Pregnant women (1) } \\
(n=32), \text { Nurses }(2) \\
(n=6)\end{array}$ & $0 / 100$ & $\begin{array}{c}1: 33.1(9.7), 2: 33.5 \\
\text { (11) }\end{array}$ & BioPatch (Chest) & HR, RR, T & Minor discomfort & $\begin{array}{c}\text { Useful for VS } \\
\text { surveillance in pregnant } \\
\text { women. Positive } \\
\text { attitudes of patients and } \\
\text { nurses }\end{array}$ \\
\hline 19 & Kim et al., 2012, USA [33] & $\begin{array}{l}\text { Comparison of CM } \\
\text { measurements } \\
\text { during physical } \\
\text { activity in extreme } \\
\text { temperatures with } \\
\text { spirometry and } \\
\text { mobile metabolic } \\
\text { system (Validation) }\end{array}$ & $\begin{array}{l}\text { Healthy individuals } \\
\qquad(n=12)\end{array}$ & $100 / 0$ & $25.5(4.1)$ & BioHarness (Chest) & $\mathrm{HR}, \mathrm{RR}$ & $\begin{array}{l}\text { Artefacts due to } \\
\text { motion and } \\
\text { perspiration }\end{array}$ & $\begin{array}{l}\text { Similar measurements } \\
\text { during exercise between } \\
\text { CM device and standard } \\
\text { methods. Correlation } \\
\text { high for HR, lower for RR }\end{array}$ \\
\hline 20 & $\begin{array}{c}\text { Van Haren et al., 2013, } \\
\text { USA [34] }\end{array}$ & $\begin{array}{l}\text { Assessment of the } \\
\text { ability of MF to } \\
\text { forecast LSI in } \\
\text { prehospital settings } \\
\text { (Cohort) }\end{array}$ & $\begin{array}{c}\text { Trauma }(n=96 \text {, No } \\
\text { LSI (1) } n=48, \text { LSI ( } 2) \\
n=48)\end{array}$ & $\begin{array}{l}\text { 1: } 88 / 12 \\
\text { 2: } 77 / 23\end{array}$ & $\begin{array}{l}\text { Overall } 48(19) \\
\quad 1: 47(18) \\
2: 49(20)\end{array}$ & $\begin{array}{l}\text { MWVSM (Forehead } \\
\text { or limb) }\end{array}$ & $\begin{array}{l}\mathrm{T}, \mathrm{SpO} 2, \mathrm{HR} \text {, pulse } \\
\text { wave transit time }\end{array}$ & $\begin{array}{l}\text { Occasionally poor } \\
\text { connection }\end{array}$ & $\begin{array}{l}\text { Useful in prehospital care } \\
\text { for trauma patients }\end{array}$ \\
\hline 21 & $\begin{array}{l}\text { Meisozo et al., 2016, } \\
\text { USA [35] }\end{array}$ & $\begin{array}{c}\text { Comparison of a CM } \\
\text { device in VS } \\
\text { surveillance with } \\
\text { standard hospital } \\
\text { equipment (Cohort) }\end{array}$ & $\begin{array}{c}\text { Trauma ICU patients } \\
\qquad(n=59)\end{array}$ & $80 / 20$ & $47(20)$ & $\begin{array}{l}\text { MWVSM (Forehead } \\
\text { or limb) }\end{array}$ & BP, T, HR, SpO2 & $\begin{array}{c}\text { Data loss; } \\
\text { under/over-triaging } \\
\text { due to signal } \\
\text { inaccuracy; requires } \\
\text { improvements }\end{array}$ & $\begin{array}{l}\text { In its current state, } \\
\text { unreliable in identifying } \\
\text { patients of highest } \\
\text { medical priority }\end{array}$ \\
\hline
\end{tabular}


Table 1. Cont.

\begin{tabular}{|c|c|c|c|c|c|c|c|c|c|}
\hline$\#$ & $\begin{array}{l}\text { First Author, Year, } \\
\text { Country, Reference }\end{array}$ & $\begin{array}{l}\text { Objective (Study } \\
\text { Type) }\end{array}$ & $\begin{array}{l}\text { Targeted Population } \\
\qquad(n)\end{array}$ & Gender (M/F, \%) & Age & $\begin{array}{l}\text { Device (Location of } \\
\text { Sensor) }\end{array}$ & $\begin{array}{l}\text { Parameter of } \\
\text { Monitoring }\end{array}$ & $\begin{array}{l}\text { Problems with } \\
\text { Devices/Barriers to } \\
\text { Implementation }\end{array}$ & Outcomes/Findings \\
\hline 22 & Dur et al., 2019, USA [36] & $\begin{array}{c}\text { Accuracy of } \\
\text { measurements and } \\
\text { quality of signal } \\
\text { (Observational) }\end{array}$ & Healthy $(n=35)$ & $54 / 46$ & $25(4)$ & $\begin{array}{l}\text { Wavelet Wristband } \\
\text { (Wrist) }\end{array}$ & HR, HRV, RR & $\begin{array}{l}\text { Quality of signal } \\
\text { influenced by } \\
\text { external aspects } \\
\text { (movements, } \\
\text { temperature, light, } \\
\text { etc.) }\end{array}$ & $\begin{array}{l}\text { Accurate measurements } \\
\text { at rest }\end{array}$ \\
\hline 23 & Li et al., 2019, USA [37] & $\begin{array}{l}\text { CM device with } \\
\text { capnography } \\
\text { (Prospective pilot) }\end{array}$ & $\begin{array}{l}\text { Respiratory patients } \\
\quad \text { in ER }(n=17)\end{array}$ & $59 / 41$ & Mean $=61$ & $\begin{array}{l}\text { Philips wearable } \\
\text { biosensor (Chest) }\end{array}$ & $\begin{array}{l}\mathrm{RR}, \mathrm{HR} \text {, ambulation, } \\
\text { posture }\end{array}$ & None reported & $\begin{array}{c}\text { CM device is comparable } \\
\text { in RR measurements } \\
\text { with capnography in ER } \\
\text { settings }\end{array}$ \\
\hline 24 & $\begin{array}{c}\text { Ordonnel et al., 2019, } \\
\text { UK [38] }\end{array}$ & $\begin{array}{c}\text { Extraction of } \\
\text { sleep-wake activity } \\
\text { data in patients of } \\
\text { various degrees of } \\
\text { disease severity } \\
\text { (Cohort) }\end{array}$ & $\begin{array}{l}\text { Heart failure (HF) } \\
\text { patients }(n=11)\end{array}$ & $36 / 64$ & $79(8.3)$ & $\begin{array}{l}\text { Proteus patch } \\
\text { (Chest) }\end{array}$ & $\begin{array}{l}\text { T, skin impedance, } \\
\text { HR, RR }\end{array}$ & $\begin{array}{l}\text { Unclear sleep-wake } \\
\text { information in } \\
\text { severe-condition } \\
\text { patients }\end{array}$ & $\begin{array}{l}\text { Feasible to monitor } \\
\text { activity during sleep and } \\
\text { wake time in HF patients }\end{array}$ \\
\hline 25 & $\begin{array}{l}\text { Hubner et al., 2015, } \\
\text { Austria [39] }\end{array}$ & $\begin{array}{l}\text { Effectiveness to } \\
\text { identify priority } \\
\text { cases (Observational } \\
\text { cross-sectional) }\end{array}$ & ER patients $(n=226)$ & $55 / 45$ & $55(43-71)$ & $\begin{array}{l}\text { Philips IntelliVue } \\
\text { Guardian Solution } \\
\text { (Chest, arm, finger) }\end{array}$ & SpO2, pulse, RR, BP & Discomfort & $\begin{array}{l}\text { Assists in identifying } \\
\text { priority patients in ER. } \\
\text { Positive attitudes. }\end{array}$ \\
\hline 26 & Liu et al., 2013, China [40] & $\begin{array}{l}\text { Evaluation of VS CM } \\
\text { at rest and during } \\
\text { exercise (Validation) }\end{array}$ & Healthy $(n=6)$ & $100 / 0$ & $22.3(3.2)$ & $\begin{array}{c}\text { EQ02 LifeMonitor } \\
\text { (many possible } \\
\text { locations) }\end{array}$ & $\begin{array}{l}\text { HR, HRV, RR, ECG, } \\
\text { RIP, body position, } \\
\text { 3-axial acceleration }\end{array}$ & $\begin{array}{l}\text { Costly due to } \\
\text { non-reusability }\end{array}$ & $\begin{array}{l}\text { Measurements are valid } \\
\text { and reliable }\end{array}$ \\
\hline 27 & $\begin{array}{l}\text { Paul et al., 2019, } \\
\text { Canada [41] }\end{array}$ & $\begin{array}{l}\text { Clinical effectiveness } \\
\text { and patient and staff } \\
\text { experiences (Pilot } \\
\text { RCT) } \\
\end{array}$ & $\begin{array}{l}\text { Surgery patients } \\
(\mathrm{I}=124, \mathrm{C}=126)\end{array}$ & $\begin{array}{l}\text { I: } 24 / 76 \\
\text { C: } 39 / 61\end{array}$ & $\begin{array}{l}\text { I: } 58.0(13.9) \\
\text { C: } 57.5(15.8)\end{array}$ & $\begin{array}{c}\text { Covidien Alarm } \\
\text { Management System } \\
\text { (finger) }\end{array}$ & SpO2, HR & $\begin{array}{l}\text { False alarms due to } \\
\text { technical issues; } \\
\text { excessive alarms in } \\
\text { tachycardic patients }\end{array}$ & $\begin{array}{l}\text { Acceptable recruitment } \\
\text { rate and positive } \\
\text { experience }\end{array}$ \\
\hline 28 & Pedone, 2013, Italy [42] & $\begin{array}{l}\text { Effectiveness of } \\
\text { telemonitoring } \\
\text { COPD patients to } \\
\text { decrease } \\
\text { hospitalizations } \\
\text { (RCT) }\end{array}$ & $\begin{array}{l}\text { Elderly COPD stage } \\
\text { II/III }(n=99 \\
\text { I }=50, C=49)\end{array}$ & $\begin{array}{l}\text { I: } 72 / 28 \\
\text { C: } 63 / 37\end{array}$ & $\begin{array}{l}\mathrm{I}=74.1(6.4) \\
\mathrm{C}=75.4(6.7)\end{array}$ & SweetAge (wrist) & $\begin{array}{l}\text { HR, physical activity, } \\
\text { T, galvanic skin } \\
\text { response }\end{array}$ & None reported & $\begin{array}{l}\text { Timely detection of state } \\
\text { deterioration to allow for } \\
\text { planned hospitalization }\end{array}$ \\
\hline 29 & Pedone, 2015, Italy [43] & $\begin{array}{l}\text { Effectiveness of } \\
\text { tele-surveillance of } \\
\text { VS (RCT) }\end{array}$ & $\begin{array}{l}\text { Elderly with HF } \\
\qquad \begin{array}{l}(n=90 \\
\mathrm{I}=47, \mathrm{C}=43)\end{array}\end{array}$ & $\begin{array}{l}\text { I: } 47 / 53 \\
\text { C }=30 / 70\end{array}$ & $\begin{array}{l}\mathrm{I}=79.9(6.8) \\
\mathrm{C}=79.7(7.8)\end{array}$ & $\begin{array}{l}\text { Sphygmomanometer, } \\
\text { a scale, a pulse } \\
\text { oximeter }\end{array}$ & $\mathrm{SpO} 2, \mathrm{HR}, \mathrm{BP}$ & None reported & $\begin{array}{l}\text { Tele-surveillance of VS } \\
\text { decreases risk of } \\
\text { hospitalization and } \\
\text { all-cause mortality in } \\
\text { elderly with HF }\end{array}$ \\
\hline 30 & Chau, 2012, China [44] & $\begin{array}{l}\text { Practicability and } \\
\text { attitudes toward } \\
\text { medical teleservices } \\
\text { (RCT) }\end{array}$ & $\begin{array}{l}\text { Elderly with COPD } \\
\text { and hospitalization } \\
\text { in the past year } \\
\qquad(n=40)\end{array}$ & $97 / 3$ & $72.93(6.04)$ & $\begin{array}{l}\text { Device kit (chest, } \\
\text { finger) }\end{array}$ & SpO2, pulse, RR & $\begin{array}{l}\text { Challenging for the } \\
\text { elderly to read small } \\
\text { screens, use multiple } \\
\text { devices, often } \\
\text { recharge battery }\end{array}$ & $\begin{array}{l}\text { Positive patient } \\
\text { perceptions }\end{array}$ \\
\hline
\end{tabular}


Table 1. Cont.

\begin{tabular}{|c|c|c|c|c|c|c|c|c|c|}
\hline$\#$ & $\begin{array}{l}\text { First Author, Year, } \\
\text { Country, Reference }\end{array}$ & $\begin{array}{l}\text { Objective (Study } \\
\text { Type) }\end{array}$ & $\begin{array}{l}\text { Targeted Population } \\
\qquad(n)\end{array}$ & Gender (M/F, \%) & Age & $\begin{array}{l}\text { Device (Location of } \\
\text { Sensor) }\end{array}$ & $\begin{array}{l}\text { Parameter of } \\
\text { Monitoring }\end{array}$ & $\begin{array}{l}\text { Problems with } \\
\text { Devices/Barriers to } \\
\text { Implementation }\end{array}$ & Outcomes/Findings \\
\hline 31 & Dellaca, 2011 Spain [45] & $\begin{array}{c}\text { Practicability of } \\
\text { continuous positive } \\
\text { airway pressure } \\
\text { (CPAP) titration at } \\
\text { home } \\
\text { (Observational) }\end{array}$ & $\begin{array}{l}\text { SAHS patients } \\
\quad(n=20)\end{array}$ & $56(3)$ & NA & $\begin{array}{l}\text { Autoset Spirit CPAP } \\
\text { machine (mask) }\end{array}$ & $\begin{array}{l}\text { Nasal pressure, } \\
\text { beathing flow and } \\
\text { air leak signals }\end{array}$ & Connection issues & $\begin{array}{l}\text { Possibility for successful } \\
\text { remote CPAP titration on } \\
\text { patients with sleep apnea } \\
\text { in home environment }\end{array}$ \\
\hline 32 & Fox, 2012 Canada [46] & $\begin{array}{l}\text { Improvement in } \\
\text { adhering to PAP } \\
\text { with telemedical } \\
\text { surveillance (RCT) }\end{array}$ & $\begin{array}{c}\text { Obstructive sleep } \\
\text { apnea patients } \\
(n=75 \\
\mathrm{I}=39, \mathrm{C}=36)\end{array}$ & $\begin{array}{l}\text { I: } 82 / 28 \\
\text { C: } 78 / 22\end{array}$ & $\begin{array}{c}53.5(11.2) \\
\text { I: } 52.0(1.8) \\
\text { C: } 55.2(11.5)\end{array}$ & $\begin{array}{l}\text { EncoreAnywhere } \\
\text { (mask) }\end{array}$ & $\begin{array}{l}\text { PAP adherence, } \\
\text { applied PAP } \\
\text { pressure, mask leak, } \\
\text { and residual } \\
\text { respiratory events }\end{array}$ & $\begin{array}{l}\text { Occasional side } \\
\text { effects }\end{array}$ & $\begin{array}{l}\text { Improved adherence to } \\
\text { PAP with telemedical } \\
\text { surveillance introduced } \\
\text { at an early stage of } \\
\text { treatment }\end{array}$ \\
\hline 33 & $\begin{array}{l}\text { Leelarathna et al., 2013, } \\
\text { UK [47] }\end{array}$ & $\begin{array}{l}\text { Evaluation of } \\
\text { glucose CM device } \\
\text { with two calibration } \\
\text { methods in critically } \\
\text { ill patients (RCT) } \\
\end{array}$ & $\begin{array}{l}\text { Patients with } \\
\text { elevated insulin } \\
\text { level }(n=24, \mathrm{I}=12, \\
\qquad \begin{array}{l}\mathrm{C}=12)\end{array}\end{array}$ & $\begin{array}{l}\text { I: } 75 / 25 \\
\text { C: } 75 / 25\end{array}$ & $\begin{array}{l}\text { I: } 62.8(16) \\
\text { C: } 58.3(12.5)\end{array}$ & $\begin{array}{l}\text { FreeStyle Navigator } \\
\text { (Subcutaneous) }\end{array}$ & $\begin{array}{l}\text { Arterial blood } \\
\text { glucose }\end{array}$ & None reported & $\begin{array}{l}\text { Accurate } \mathrm{CM} \text { of glucose, } \\
\text { may be useful for } \\
\text { intensive insulin therapy }\end{array}$ \\
\hline 34 & $\begin{array}{c}\text { Lockman et al., 2011, } \\
\text { USA [9] }\end{array}$ & $\begin{array}{c}\text { Identifying } \\
\text { tonic-clonic seizures } \\
\text { with a CM device } \\
\text { (Cohort) }\end{array}$ & $\begin{array}{c}\text { Epilepsy patients } \\
\quad(n=40 \\
\text { seizures }=6)\end{array}$ & Seizures: 50/50 & $31(23-48)$ & $\begin{array}{l}\text { SmartWatch (wrist } \\
\quad \text { or ankle) }\end{array}$ & $\begin{array}{l}\text { Rhythmic, repetitive } \\
\text { movement of an } \\
\text { extremity }\end{array}$ & Battery; connection & $\begin{array}{l}\text { Measurements } \\
\text { comparable to those of } \\
\text { standard equipment }\end{array}$ \\
\hline & & $\begin{array}{l}\text { reviation: BP-Bloo } \\
\text { sure; ECG-Electro } \\
\text {-Life Saving Interv } \\
\text { trolled Trial; RIP-I }\end{array}$ & $\begin{array}{l}\text { ressure; BR-Breath } \\
\text { diogram; ER-Eme } \\
\text { tion; MAP-Mean } \\
\text { piratory Inductance }\end{array}$ & $\begin{array}{l}\text { Rate, C-Contro } \\
\text { ncy Room; HF- } \\
\text { erial Pressure; } M \\
\text { ethysmography, }\end{array}$ & $\begin{array}{l}\text {-Continuous I } \\
\text { Gailure; HR- } \\
\text {-Modified Ea } \\
\text {-Sleep Apnea }\end{array}$ & $\begin{array}{l}\text { toring; COPD_Chrc } \\
\text { t Rate, I-Interventi } \\
\text { Jarning Score; MF- } \\
\text { oopnea Syndrome; S }\end{array}$ & $\begin{array}{l}\text { c obstructive pulmo } \\
\text {; ICU-Intensive C } \\
\text { Iurphy Factor, PAP- } \\
\text { D2-Oxygen Satura }\end{array}$ & $\begin{array}{l}\text { ry disease, CPAP- } \\
\text { Unit; RR-Respir } \\
\text { ?ositive Airway Pr } \\
\text { n; VS-Vital Signs. }\end{array}$ & $\begin{array}{l}\text { hinuous Positive Airwa } \\
\text { y Rate, T-Temperatur } \\
\text { ure; RCT-Randomize }\end{array}$ \\
\hline
\end{tabular}




\subsection{Medical Professional-Related Concerns}

1. Nurses were concerned that the devices would replace them $[1,7,15]$. 2. The high volume of data received from the monitoring devices can lead to an increased workload on personnel and could lead to personnel withholding themselves from checking these data, resulting in a diminution of the predictive value of continuous monitoring [4]. 3. A high quantity of redundant audible alerts interrupts nursing work and apparently reduces patient safety $[11,54]$. 4. Additional training for caregivers and healthcare professionals might be required (study coordinators requested to gain additional experience with devices and their software to increase their comfort in managing these devices) [55]. 5. Unnecessary false alarms may lead to the loss of attention of healthcare providers and caregivers to patients $[2,12,16]$. 6 . The personnel did not always identify a deterioration pattern [3].

\section{Discussion}

This systematic review summarizes the evidence related to the application of remote monitoring of chronically critically ill patients after hospital discharge. Although there is an insufficient amount of evidence related to the home-based management of these patients using advanced technologies, we summarized the evidence from other areas of remote monitoring that can be used for this group of patients. Multisystem dysfunction, including cerebral, cognitive, and behavioral impairment is present in almost all patients with chronic critical illness. Most patients have functional impairment, which requires close observation and involvement of caregivers $[5,7,8,10,51,56,57]$. Therefore, the remote monitoring systems might be valuable for this patient population.

\subsection{General Rationale of Using Remote Patient Monitoring}

Remote monitoring systems include a monitor/terminal for the end-user, a communication network, a data acquisition system, and a data processing system. The incentives for remote monitoring include real-time and continuous tracking of symptoms, early detection of complications and deterioration of vital function, lower treatment costs, and the ability to activate an emergency response, if needed [55]. Implementation of remote patient monitoring can improve the quality of patient management, reduce complication and deterioration rates, and decrease the burden on family by likely decreasing healthcare costs through catching and responding to complications and deteriorations as early as possible.

Innovations in digital medicine are revolutionizing healthcare delivery and are significantly changing the interaction of healthcare providers with patients by developing and expanding the functions of monitoring devices [53,58]. The development of monitoring devices has led to opportunities for remote monitoring of clinically important physiological variables outside of hospital settings [59]. Such devices can be implemented into routine management of chronic medical conditions and can provide useful information for both medical personnel and patients $[58,60]$. A lot of attention has been given to the applications of wearable body sensors for remote monitoring [61].

A wide variety of sensors can be incorporated into smart devices to allow for the remote monitoring of the most appropriate variables and for data transmission. These sensors can measure numerous variables including vital signs (blood pressure, respiratory rate, temperature, level of consciousness, blood oxygen saturation, blood glucose level) and body movement. The sensors can be implanted in body parts and clothing, or subcutaneously. They are becoming more reliable, accurate, and easy to use for patient monitoring [62].

Such technologies can be used for continuous monitoring, prediction, prevention, diagnosis, and treatment of pathological events in chronic critical illness. Despite considerable advancement in this area, the widespread use of this technology in chronic critical illness remains very limited. The most important types of sensors that can be useful for such patients after hospital discharge can give measurements on hemoglobin oxygen saturation, heart rate, respiratory rate, ECG, blood glucose concentration, body temperature, posture 
and movement, vibrations, and coughing events. The wearable devices can be worn in several anatomical locations, such as the chest, arm, leg, waist, and wrist [58].

The most broadly accepted directions of remote monitoring include patient reported outcomes, telemonitoring, and quantifying self-hybrid models [50].

The applications of wearable sensors in the management of chronically critically ill patients might be useful away from the hospital. These sensors may reduce the length of hospital stay, cost of hospitalization, and hospital bed turnover. In turn, the use of wearable technologies might also improve the quality of patient care in non-hospital settings, reduce family burden, and likely prolong the life span and the quality of rehabilitation, reducing the risk of complications and readmission to hospitals.

Remote monitoring has been shown to increase the quality of care in cardiovascular patients [63]. Moreover, there is a high demand for device-driven detection of breathing patterns, respiratory rate, and fatal respiratory disorders [63]. The ring sensor devices have been used to improve the management of congestive heart failure and hypertension $[58,64]$. Vital sign monitoring is the most important type of monitoring that can measure a wide variety of parameters, ranging from electrical to biochemical signs [65].

\subsection{Glucose Monitoring}

Diabetes has been consistently reported as one of the most prevalent chronic conditions in chronic critically ill patients. To decrease the risk of further complications, strict glucose control is required. Blood glucose levels, especially in chronically ill patients with diabetes, can fluctuate significantly throughout the day and, therefore, may require multiple daily measurements. Traditional glucose control based on blood sample collection through a finger-prick is an invasive, inconvenient method [66]. The application of wearable sensors for measuring blood glucose levels could improve the quality of diabetes management and patients' autonomy [47]. Wearable sensors with the function of continuous glucose monitoring have been successfully used to reduce hemoglobin A1c $(\mathrm{HbA} 1 \mathrm{c})$, improving the quality of life and health outcomes of patients [65]. The medium and long length electrodes penetrate into the deep layers of the tissue and give data related to fluctuations in glucose levels [58].

\subsection{Remote Neurological Monitoring}

Remote neurological monitoring plays an important role in the management of chronically critically ill patients, especially in outpatient postoperative management and rehabilitation. Several neurological parameters can be controlled using remote monitoring devices. A wristwatch has been shown to detect seven out of eight seizure episodes and accurately transfer related information to the caregiver [9]. While this device does not predict or treat convulsions, it can alert the caregiver quickly, reducing the risk of serious damage and death [58]. Although remote monitoring devices have been used for a wide variety of diseases and conditions, high-quality studies regarding chronic critical illness are missing. Given the progressively increasing number of such patients, it might be reasonable to implement these technologies in chronically critical ill patients after hospital discharge.

\subsection{Limitations of This Study}

One of the most important limitations of this systematic review is the high heterogeneity of the reported data. Since most of the included studies were performed by researchers with technical majors (engineering, computer sciences), most of them focused on technical characteristics, and not all studies reported the performance data that would help to generate clinical evidence (sensitivity, specificity, positive and negative predictive data). Therefore, we did not perform a meta-analysis. Despite this, we found it useful to transfer the existing knowledge from technical to medical fields. Another limitation is that since the definition of chronic critical illness is not widely used in clinical practice, the number of studies specifically focusing on chronic critical illness patients after hospital discharge is very limited. There was not enough available evidence to conduct the subgroup analysis, 
e.g., in some studies the sample size was too small, in some studies there were too many comparisons undertaken, and some other studies either did not characterize the patient population or did not subdivide them into groups at all. We did not break down the study sample of the secondary data into subgroups, due to the reason that we might end up with too few participants in each group to detect differences, or to ensure that differences were not a matter of chance. Therefore, we focused on the general issues and potential solutions in the management of chronic critically ill patients after the discharge from the hospital without subdividing the cohort into several groups.

Some of these limitations can be overcome by collaborating with medical doctors, clinical investigators, nurses, caregivers, engineers, and information technology professionals. This collaboration can minimize the existing gap and make clinical trials in this area available. Only successful clinical trials can lead towards a wide implementation of these technologies in clinical practice.

\subsection{Future Research}

It is expected that advanced wearable technologies will continue to evolve. We need to ensure that we will be able to provide appropriate care to CCI patients. There is not enough attention from both the healthcare and scientific worlds toward this problem. The responsibility of healthcare, science, and technology is to make the lives of patients easier and improve their quality. Chronic critical illness is a healthcare and societal issue that could be potentially solved by creating a special services and involving modern technologies.

The implementation of remote-sensor based monitoring technologies can bring the following benefits to the existing healthcare system: 1 . Reduce the length of hospital stays; 2. Reduce healthcare spending; 3 . Reduce the burden on families and healthcare providers during the post-hospital discharge period; 4 . Improve the quality of life of patients as well as their family members; 5 . Enhance research and innovation; 6. Improve the personalization of medical care; 7. Enhance the adoption of these monitoring technologies by healthcare providers and patients.

The feasibility of monitoring activity patterns of patients with very severe forms of their conditions warrants further research [64]. Apparently, there are considerable economic investments devoted to pharmacologic rather than technological innovation [9]. Continuous patient monitoring systems can be successfully accepted, implemented, and used only if they improve efficiency in identifying patient destabilization and if they do not increase the workload of healthcare providers. Therefore, more studies are needed to address this issue.

\section{Conclusions}

Given the current progress in intensive care medicine, the prevalence of chronic critical illness will continue to grow, leading to an increase in family burden, healthcare utilization, and economic costs. The main issues in the management of these patients are: the shortage of caretakers, the episodic nature of vital function monitoring (e.g., episodic measuring blood pressure leads to missing important critical events such as hypertension, hypotension, and hypoxia), and failure to catch and manage critical physiological events at the right time which can finally result in poor outcomes. Wearable devices can be used to monitor blood pressure, heart rate, pulse, respiratory rate, blood oxygen saturation, metabolism, and central nervous system function. While numerous studies have been conducted, there are still many questions to be answered. The most important of these revolve around the performance of the remote monitoring systems (sensitivity, specificity, positive predictive value, and negative predictive value), safety, clinical and economic outcomes, and acceptance of the devices by patients, caretakers, and healthcare professionals. Future clinical trials are warranted to investigate the value of remote monitoring in the management of critically ill patients. 
Author Contributions: Conceptualization: D.V., E.S., R.B., Y.A. and F.B.; methodology D.V., R.B., R.B. and F.B.; data curation: D.V. and M.A.; writing-original draft preparation: D.V.; writing-review and editing: D.V., Y.A., M.A., R.B. and F.B.; supervision: F.B. All authors have read and agreed to the published version of the manuscript.

Funding: The authors received no financial support for the research, authorship and/or publication of this article.

\section{Institutional Review Board Statement: Not applicable.}

Informed Consent Statement: Not applicable.

Conflicts of Interest: The authors declare no conflict of interest.

\section{References}

1. Nelson, J.E.; Cox, C.E.; Hope, A.A.; Carson, S.S. Chronic critical illness. Am. J. Respir. Crit. Care Med. 2010, 182, 446-454. [CrossRef]

2. Girard, K.; Raffin, T.A. The chronically critically ill: To save or let die? Respir. Care 1985, 30, 339-347.

3. Zilberberg, M.D.; Luippold, R.S.; Sulsky, S.; Shorr, A.F. Prolonged acute mechanical ventilation, hospital resource utilization, and mortality in the United States. Crit. Care Med. 2008, 6, 724-730. [CrossRef]

4. Zilberberg, M.D.; De Wit, M.; Shorr, A.F. Accuracy of previous estimates for adult prolonged acute mechanical ventilation volume in 2020: Update using 2000-2008 data. Crit. Care Med. 2012, 40, 18-20. [CrossRef]

5. Douglas, S.L.; Daly, B.J.; Brennan, P.F.; Gordon, N.H.; Uthis, P. Hospital readmission among long-term ventilator patients. Chest 2001, 120, 1278-1286. [CrossRef]

6. Nasraway, S.A.; Button, G.J.; Rand, W.M.; Hudson-Jinks, T.; Gustafson, M. Survivors of catastrophic illness: Outcome after direct transfer from intensive care to extended care facilities. Crit. Care Med. 2000, 28, 19-25. [CrossRef]

7. Nelson, J.E.; Tandon, N.; Mercado, A.F.; Camhi, S.L.; Ely, E.W.; Morrison, R.S. Brain dysfunction: Another burden for the chronically critically ill. Arch. Intern. Med. 2006, 166, 1993-1999. [CrossRef]

8. Van den Berghe, G.; De Zegher, F.; Veldhuis, J.D.; Wouters, P.; Gouwy, S.; Stockman, W.; Weekers, F.; Schetz, M.; Lauwers, P.; Bouillon, R.; et al. Thyrotrophin and prolactin release in prolonged critical illness: Dynamics of spontaneous secretion and effects of growth hormone-secretagogues. Clin. Endocrinol. 1997, 47, 599-612. [CrossRef]

9. Lockman, J.; Fisher, R.S.; Olson, D.M. Detection of seizure-like movements using a wrist accelerometer. Epilepsy Behav. 2011, 20, 638-664. [CrossRef]

10. Mills, S. Electronic Health Records and Use of Clinical Decision Support. Crit. Care Nurs. Clin. N. Am. 2019, 31, 125-131. [CrossRef]

11. Macintyre, N.R. Chronic critical illness: The growing challenge to health care. Respir. Care 2012, 57, 1021-1027. [CrossRef]

12. Vegesna, A.; Tran, M.; Angelaccio, M.; Arcona, S. Remote Patient Monitoring via Non-Invasive Digital Technologies: A Systematic Review. Telemed. J. E Health 2017, 23, 3-17. [CrossRef] [PubMed]

13. Available online: http:/ / www.prisma-statement.org/PRISMAStatement/ (accessed on 10 February 2022).

14. Available online: https://www.crd.york.ac.uk/prospero/ (accessed on 10 February 2022).

15. Prgomet, M.; Cardona-Morrell, M.; Nicholson, M.; Lake, R.; Long, J.; Westbrook, J.; Braithwaite, J.; Hillman, K. Vital signs monitoring on general wards: Clinical staff perceptions of current practices and the planned introduction of continuous monitoring technology. Int. J. Qual. Health Care 2016, 28, 515-521. [CrossRef]

16. Weller, R.S.; Foard, K.L.; Harwood, T.N. Evaluation of a wireless, portable, wearable multi-parameter vital signs monitor in hospitalized neurological and neurosurgical patients. J. Clin. Monit. Comput. 2018, 32, 945-951. [CrossRef]

17. Verrillo, S.C.; Cvach, M.; Hudson, K.W.; Winters, B.D. Using Continuous Vital Sign Monitoring to Detect Early Deterioration in Adult Postoperative Inpatients. J. Nurs. Care Qual. 2019, 34, 107-113. [CrossRef] [PubMed]

18. Weenk, M.; Van Goor, H.; Frietman, B.; Engelen, L.J.; van Laarhoven, C.J.; Smit, J.; Bredie, S.J.; van de Belt, T.H. Continuous Monitoring of Vital Signs Using Wearable Devices on the General Ward: Pilot Study. JMIR mHealth uHealth 2017, 5, e91. [CrossRef] [PubMed]

19. Watkins, T.; Whisman, L.; Booker, P. Nursing assessment of continuous vital sign surveillance to improve patient safety on the medical/surgical unit. J. Clin. Nurs. 2016, 25, 278-281. [CrossRef]

20. Downey, C.; Randell, R.; Brown, J.; Jayne, D.G. Continuous Versus Intermittent Vital Signs Monitoring Using a Wearable, Wireless Patch in Patients Admitted to Surgical Wards: Pilot Cluster Randomized Controlled Trial. J. Med. Internet Res. 2018, 20 , e10802. [CrossRef]

21. Downey, C.L.; Brown, J.M.; Jayne, D.G.; Randell, R. Patient attitudes towards remote continuous vital signs monitoring on general surgery wards: An interview study. Int. J. Med. Inform. 2018, 114, 52-56. [CrossRef]

22. Hernandez-Silveira, M.; Ahmed, K.; Ang, S.; Zandari, F.; Mehta, T.; Weir, R.; Burdett, A.; Toumazou, C.; Brett, S.J. Assessment of the feasibility of an ultra-low power, wireless digital patch for the continuous ambulatory monitoring of vital signs. BMJ Open 2015, 5, e006606. [CrossRef] 
23. Hernandez-Silveira, M.; Wieczorkowski-Rettinger, K.; Ang, S.; Burdetta, A. Preliminary assessment of the SensiumVitals ${ }^{\circledR}$ : A low-cost wireless solution for patient surveillance in the general wards. Annu. Int. Conf. IEEE Eng. Med. Biol. Soc. 2015, 2015, 4931-4937. [CrossRef] [PubMed]

24. Downey, C.; Ng, S.; Jayne, D.; Wong, D. Reliability of a wearable wireless patch for continuous remote monitoring of vital signs in patients recovering from major surgery: A clinical validation study from the TRaCINg trial. BMJ Open 2019, 9, e031150. [CrossRef] [PubMed]

25. Chan, A.M.; Selvaraj, N.; Ferdosi, N.; Narasimhan, R. Wireless patch sensor for remote monitoring of heart rate, respiration, activity, and falls. Annu. Int. Conf. IEEE Eng. Med. Biol. Soc. 2013, 2013, 6115-6118. [CrossRef] [PubMed]

26. Izmailova, E.S.; McLean, I.L.; Bhatia, G. Evaluation of Wearable Digital Devices in a Phase I Clinical Trial. Clin. Transl. Sci. 2019, 12, 247-256. [CrossRef] [PubMed]

27. Breteler, M.J.M.; Huizinga, E.; Van Loon, K.; Leenen, L.P.; Dohmen, D.A.; Kalkman, C.J.; Blokhuis, T.J. Reliability of wireless monitoring using a wearable patch sensor in high-risk surgical patients at a step-down unit in the Netherlands: A clinical validation study. BMJ Open 2018, 8, e020162. [CrossRef]

28. Selvaraj, N.; Nallathambi, G.; Moghadam, R.; Aga, A. Fully Disposable Wireless Patch Sensor for Continuous Remote Patient Monitoring. Annu. Int. Conf. IEEE Eng. Med. Biol. Soc. 2018, 2018, 1632-1635. [CrossRef]

29. Liu, N.T.; Holcomb, J.B.; Wade, C.E.; Darrah, M.I.; Salinas, J. Evaluation of standard versus nonstandard vital signs monitors in the prehospital and emergency departments: Results and lessons learned from a trauma patient care protocol. J. Trauma Acute Care Surg. 2014, 77, S121-S126. [CrossRef]

30. Liu, N.T.; Holcomb, J.B.; Wade, C.E.; Darrah, M.I.; Salinas, J. Data quality of a wearable vital signs monitor in the pre-hospital and emergency departments for enhancing prediction of needs for life-saving interventions in trauma patients. J. Med. Eng. Technol. 2015, 39, 316-321. [CrossRef]

31. Razjouyan, J.; Grewal, G.S.; Rishel, C.; Parthasarathy, S.; Mohler, J.; Najafi, B. Activity Monitoring and Heart Rate Variability as Indicators of Fall Risk: Proof-of-Concept for Application of Wearable Sensors in the Acute Care Setting. J. Gerontol. Nurs. 2017, 43, 53-62. [CrossRef]

32. Boatin, A.A.; Wylie, B.J.; Goldfarb, I.; Azevedo, R.; Pittel, E.; Ng, C.; Haberer, J.E. Wireless Vital Sign Monitoring in Pregnant Women: A Functionality and Acceptability Study. Telemed. J. E Health 2016, 22, 564-571. [CrossRef]

33. Kim, J.H.; Roberge, R.; Powell, J.B.; Shafer, A.B.; Williams, W.J. Measurement accuracy of heart rate and respiratory rate during graded exercise and sustained exercise in the heat using the Zephyr BioHarness. Int. J. Sports Med. 2013, 34, 497-501. [CrossRef] [PubMed]

34. Van Haren, R.M.; Thorson, C.M.; Valle, E.J.; Busko, A.M.; Jouria, J.M.; Livingstone, A.S.; Namias, N.; Schulman, C.I.; Proctor, K.G. Novel prehospital monitor with injury acuity alarm to identify trauma patients who require lifesaving intervention. J. Trauma Acute Care Surg. 2014, 76, 743-749. [CrossRef] [PubMed]

35. Meizoso, J.P.; Allen, C.J.; Ray, J.J.; Van Haren, R.M.; Teisch, L.F.; Baez, X.R.; Livingstone, A.S.; Namias, N.; Schulman, C.I.; Proctor, K.G. Evaluation of Miniature Wireless Vital Signs Monitor in a Trauma Intensive Care Unit. Mil. Med. 2016, 181, 199-204. [CrossRef] [PubMed]

36. Dur, O.; Rhoades, C.; Ng, M.S.; Elsayed, R.; Van Mourik, R.; Majmudar, M.D. Design Rationale and Performance Evaluation of the Wavelet Health Wristband: Benchtop Validation of a Wrist-Worn Physiological Signal Recorder. JMIR $m$ Health uHealth 2018, 6, e11040. [CrossRef] [PubMed]

37. Li, T.; Divatia, S.; McKittrick, J.; Moss, J.; Hijnen, N.M.; Becker, L.B. A pilot study of respiratory rate derived from a wearable biosensor compared with capnography in emergency department patients. Open Access Emerg. Med. 2019, 11, 103-108. [CrossRef]

38. OrDonnell, J.; Velardo, C.; Shah, S.A.; Khorshidi, G.S.; Salvi, D.; Rahimi, K.; Tarassenko, L. Physical Activity and Sleep Analysis of Heart Failure Patients using Multi-sensor Patches. Annu. Int. Conf. IEEE Eng. Med. Biol. Soc. 2018, 2018, 6092-6095. [CrossRef] [PubMed]

39. Hubner, P.; Schober, A.; Sterz, F.; Stratil, P.; Wallmueller, C.; Testori, C.; Grassmann, D.; Lebl, N.; Ohrenberger, I.; Herkner, H.; et al. Surveillance of Patients in the Waiting Area of the Department of Emergency Medicine. Medicine 2015, 94, e2322. [CrossRef]

40. Liu, Y.; Zhu, S.H.; Wang, G.H.; Ye, F.; Li, P.Z. Validity and reliability of multiparameter physiological measurements recorded by the Equivital LifeMonitor during activities of various intensities. J. Occup. Environ. Hyg. 2013, 10, 78-85. [CrossRef]

41. Paul, J.E.; Chong, M.A.; Buckley, N.; Harsha, P.; Shanthanna, H.; Tidy, A.; Buckley, D.; Clarke, A.; Young, C.; Wong, T.; et al. Vital sign monitoring with continuous pulse oximetry and wireless clinical notification after surgery (the VIGILANCE pilot study)-a randomized controlled pilot trial. Pilot Feasibility Stud. 2019, 5, 36. [CrossRef]

42. Pedone, C.; Chiurco, D.; Scarlata, S.; Incalzi, R.A. Efficacy of multiparametric telemonitoring on respiratory outcomes in elderly people with COPD: A randomized controlled trial. BMC Health Serv. Res. 2013, 13, 82. [CrossRef]

43. Pedone, C.; Rossi, F.F.; Cecere, A.; Costanzo, L.; Incalzi, R.A. Efficacy of a Physician-Led Multiparametric Telemonitoring System in Very Old Adults with Heart Failure. J. Am. Geriatr. Soc. 2015, 63, 1175-1180. [CrossRef] [PubMed]

44. Chau, J.P.C.; Lee, D.T.Z.; Yu, D.S.F. A feasibility study to investigate the acceptability and potential effectiveness of a telecare service for older people with chronic obstructive pulmonary disease. Int. J. Med. Inform. 2012, 81, 674-682. [CrossRef] [PubMed]

45. Dellacà, R.; Montserrat, J.M.; Govoni, L.; Pedotti, A.; Navajas, D.; Farré, R. Telemetric CPAP titration at home in patients with sleep apnea-hypopnea syndrome. Sleep Med. 2011, 12, 153-157. [CrossRef] 
46. Fox, N.; Hirsch-Allen, A.J.; Goodfellow, E.; Wenner, J.; Fleetham, J.; Ryan, C.F.; Kwiatkowska, M.; Ayas, N.T. The impact of a telemedicine monitoring system on positive airway pressure adherence in patients with obstructive sleep apnea: A randomized controlled trial. Sleep 2012, 35, 477-481. [CrossRef]

47. Leelarathna, L.; English, S.W.; Thabit, H.; Caldwell, K.; Allen, J.M.; Kumareswaran, K.; Wilinska, M.E.; Nodale, M.; Haidar, A.; Evans, M.L.; et al. Accuracy of subcutaneous continuous glucose monitoring in critically ill adults: Improved sensor performance with enhanced calibrations. Diabetes Technol. Ther. 2014, 16, 97-101. [CrossRef] [PubMed]

48. Kalb, T.H.; Lorin, S. Infection in the chronically critically ill: Unique risk profile in a newly defined population. Crit. Care Clin. 2002, 18, 529-552. [CrossRef]

49. Van den Berghe, G.; de Zegher, F.; Veldhuis, J.D.; Wouters, P.; Awouters, M.; Verbruggen, W.; Schetz, M.; Verwaest, C.; Lauwers, P.; Bouillon, R.; et al. The somatotropic axis in critical illness: Effect of continuous growth hormone (GH)-releasing hormone and GH-releasing peptide-2 infusion. J. Clin. Endocrinol. Metab. 1997, 82, 590-599. [CrossRef]

50. Appelboom, G.; Sussman, E.S.; Raphael, P.; Juillière, Y.; Reginster, J.Y.; Connolly, E.S. A critical assessment of approaches to outpatient monitoring. Curr. Med. Res. Opin. 2014, 30, 1383-1384. [CrossRef]

51. Engoren, M.; Arslanian-Engoren, C.; Fenn-Buderer, N. Hospital and long-term outcome after tracheostomy for respiratory failure. Chest 2004, 125, 220-227. [CrossRef] [PubMed]

52. Scheinhorn, D.J.; Hassenpflug, M.S.; Votto, J.J.; Chao, D.C.; Epstein, S.K.; Doig, G.S.; Knight, E.B.; Petrak, R.A.; Ventilation Outcomes Study Group. Post-ICU mechanical ventilation at 23 long-term care hospitals: A multicenter outcomes study. Chest 2007, 131, 85-93. [CrossRef]

53. Ricciardi, L.; Mostashari, F.; Murphy, J.; Daniel, J.G.; Siminerio, E.P. A national action plan to support consumer engagement via e-health. Health Aff. 2013, 32, 376-384. [CrossRef]

54. Graham, K.C.; Cvach, M. Monitor alarm fatigue: Standardizing use of physiological monitors and decreasing nuisance alarms. Am. J. Crit. Care 2010, 19, 28-34. [CrossRef]

55. Malasinghe, L.P.; Ramzan, N.; Dahal, K. Remote patient monitoring: A comprehensive study. J. Ambient Intell. Human. Comput. 2019, 10, 57-76. [CrossRef]

56. Miller, E.; Polson, D. Apps, Avatars and Robots: The Future of Mental Healthcare. Issues Ment. Health Nurs. 2019, 40, 208-214. [CrossRef]

57. Sanchez-Morillo, D.; Fernandez-Granero, M.A.; Leon-Jimenez, A. Use of predictive algorithms in-home monitoring of chronic obstructive pulmonary disease and asthma: A systematic review. Chron. Respir. Dis. 2016, 13, 264-283. [CrossRef]

58. Appelboom, G.; Camacho, E.; Abraham, M.E.; Bruce, S.S.; Dumont, E.L.; Zacharia, B.E.; D’Amico, R.; Slomian, J.; Reginster, J.Y.; Bruyère, O.; et al. Smart wearable body sensors for patient self-assessment and monitoring. Arch. Public Health 2014, 72, 28. [CrossRef]

59. Hayakawa, M.; Uchimura, Y.; Omae, K.; Waki, K.; Fujita, H.; Ohe, K. A smartphone-based medication self-management system with realtime medication monitoring. Appl. Clin. Inform. 2013, 4, 37-52. [CrossRef]

60. Chen, K.Y.; Bassett, D.R. The technology of accelerometry-based activity monitors: Current and future. Med. Sci. Sports Exerc. 2005, 37, S490-S500. [CrossRef]

61. Swan, M. Emerging patient-driven health care models: An examination of health social networks, consumer personalized medicine and quantified self-tracking. Int. J. Environ. Res. Public Health 2009, 6, 492-525. [CrossRef]

62. Cook, D.J.; Thompson, J.E.; Prinsen, S.K.; Dearani, J.A.; Deschamps, C. Functional recovery in the elderly after major surgery: Assessment of mobility recovery using wireless technology. Ann. Thorac. Surg. 2013, 96, 1057-1061. [CrossRef]

63. Chandrasekaran, V. Measuring Vital Signs Using Smart Phones; UNT Digital Library. 2010. Available online: https://digital. library.unt.edu/ark:/67531/metadc33139/m1/1/ (accessed on 10 February 2022).

64. Chan, M.; Estève, D.; Fourniols, J.Y.; Escriba, C.; Campo, E. Smart wearable systems: Current status and future challenges. Artif. Intell. Med. 2012, 56, 137-156. [CrossRef]

65. Rodbard, D. Continuous Glucose Monitoring: A Review of Successes, Challenges, and Opportunities. Diabetes Technol. Ther. 2016, 18, S3-S13. [CrossRef]

66. Yoo, E.H.; Lee, S.Y. Glucose biosensors: An overview of use in clinical practice. Sensors 2010, 10, 4558-4576. [CrossRef] 